\title{
Working memory and L2 development across the lifespan: a commentary
}

Book or Report Section

Accepted Version

Wright, C. (2015) Working memory and L2 development across the lifespan: a commentary. In: Wen, Z. (E.), Mota, M. B. and McNeill, A. (eds.) Working memory in second language acquisition and processing. Multilingual Matters, Bristol, pp. 285-298. ISBN 9781783093588 Available at http://centaur.reading.ac.uk/49729/

It is advisable to refer to the publisher's version if you intend to cite from the work. See Guidance on citing.

Publisher: Multilingual Matters

All outputs in CentAUR are protected by Intellectual Property Rights law, including copyright law. Copyright and IPR is retained by the creators or other copyright holders. Terms and conditions for use of this material are defined in the End User Agreement. 


\section{www.reading.ac.uk/centaur}

\section{CentAUR}

Central Archive at the University of Reading

Reading's research outputs online 


\section{WM and L2 development across the life span: A commentary Clare Wright}

As the contributions to this book clearly demonstrate, the exciting implications and applications of WM research for L2 development have become clearly demonstrated since the early studies of Juffs, Service and others in the 1990s. Understanding the complex nature of WM's involvement in SLA however remains challenging across a range of theoretical and empirical dimensions, as shown throughout this volume, and particularly in Mitchell, Jarvis, O'Malley and Konstantinova's chapter. As Mitchell et al. showed, some of the often contradictory findings from WM/SLA research may arise from fundamental problems in research design. WM tests themselves are not always reliably used as language-independent measures, which can produce a confounding effect of proficiency on WM scores if measured in the L2. Mitchell et al, and other authors throughout this volume, also highlight the need to distinguish between the different WM constructs such as phonological short term memory (PSTM) and executive working memory (EWM). Then as Mitchell et al, and others, point out, SLA researchers also need to establish a robust logic for why each construct may apply to different aspects of language learning, rather than apply a general assumption of WM to L2 proficiency, without a clear identification of what is meant by proficiency. Mitchell et al provide a useful introduction to thinking through the implications of how WM may work in different ways at different stages of language development - suggesting that PSTM may benefit earlier-stage learners, while EWM is associated with advanced learners.

This chapter seeks to reflect on Mitchell et al's work within the wider context of SLA and WM research, and in particular the claims of different effects of different aspects of WM as learners progress through stages of learning. Some of the challenges for SLA will be presented arising from the theoretical and empirical evidence of WM across the L2 lifespan, and the chapter finishes with implications and suggestions for where we can go from here.

\section{Definitions of WM}

It is worth starting off with some caveats over definitions and models of WM used in this chapter. Much SLA research assumes that language learning shares similarities with skill development, shifting with enough practice from slow, controlled, consciously manipulated cognitive processes towards faster, automatic, unconscious processes. These two ends of the language learning spectrum are associated with constructs borrowed from information 
processing and psycholinguistics - the explicit/implicit learning distinction (R. Ellis et al. 2009), and the declarative/procedural distinction (Ullman, 2004). Space precludes a detailed discussion here (but see, for example, N. Ellis, 2005; Han \& Finneran, 2013; Hulstijn, 2005). As such, WM is most usually associated with handling processes that involve controlled, conscious manipulation or attention (Levelt, 1989, 1993), which is especially the case in L2 production until late stages of highly advanced automatised proficiency (Kormos, 2006). WM is therefore typically investigated for its involvement in language uses that benefit from consciously controlled explicit processes - such as vocabulary learning, or reading and interpreting ambiguous material that makes implicit automatic processing break down (Juffs \& Harrington, 1995). This fits well into research questions that look at the role of WM for learners in the pre-automatic stage of learning, where it is clear they are noticing, monitoring and using other explicit, consciously attended processes to control their language interpretation and production. However, the investigation of the involvement of WM in such learning contexts has not always distinguished between PSTM and EWM. But in fact in strict psycholinguistic terms, many of the complex language activities involving various aspects of WM, particularly EWM, must take place at pre-conscious levels of processing (Jackendoff, 2002). Therefore it is worth carefully distinguishing between PSTM and EWM to clarify how they may also be involved in non-explicit processing, as procedural WM (Oberauer, 2010). This theoretical discussion of the nature of the broad scope of WM will need to be looked at in future research, at all stages of the life-span. For now, to fit the scope of this chapter, I will assume the usual definition of PSTM and EWM as primarily assisting consciously monitored processing, including of explicitly learned, declarative linguistic knowledge. The interesting question addressed by Mitchell et al, and reviewed here, is how far PSTM and EWM may play a differential role at various stages of the learning process, and across the lifespan.

\section{WM in the earliest years of linguistic development}

As many children grow up bilingual, or start learning a second language from very young ages, it is logical to try and tease out how early in the lifespan WM (including PSTM) may be seen to have an effect on SLA. Traditionally, it was seen that infant learning was largely procedural or implicit, with little storage of explicit linguistic information beyond very simple words, and little capacity for executive control over declarative or explicit memory. WM was therefore assumed to have no major role in the earliest stages of first language acquisition, before about 3-4 years of age (Baddeley et al., 2009). However, more 
sophisticated types of testing have now shown that even by one year old, infants show evidence of explicit memory, remembering and applying learned information (ibid), so this assumption may need to be revised, particularly in bilingual children. Very recent research into executive control in children suggests that there are effects of WM control which favour bilingual children even as young as 24 months (Poulin-Dubois et al., 2011).

Most of the research on WM and children starts around 4 years of age upwards, when both storage and processing capacities begin to be identifiable using standard tests. The main area of work, to date, mainly in the US and the UK, has focused on Baddeley's multicomponent model. Standardised tests for this age group are widely used to test phonological short-term memory, or PSTM (Gathercole \& Baddeley, 1993), but often now also include an executive working memory (EWM) component (Alloway \& Gathercole 2005). PSTM shows steady increase in average item storage (including chunks of linked items) from around two items at the age of five years, to an adult average of six items by around 15-20 years, before starting to decline in middle-age, and particularly after around 65 years (Alloway, 2011). Correlating this storage capacity with a range of school activities has found robust significant associations between PSTM and vocabulary development in children. Similarly, as executive control develops, EWM effects have been found on reading comprehension and amount of information produced in story telling tasks (Adams \& Gathercole, 1996, 2000), as well as in spoken narrative comprehension (Montgomery et al., 2009). There are identifiable effects in adults too, where PSTM has been identified as connected with novel word learning (Papagno \& Vallar, 1995); in addition, the well-known WM effect on better reading comprehension (Daneman \& Carpenter, 1980) has been found also in successful judgements on semantic ambiguity and syntactic complexity, such as in "garden-path" sentence resolution (King \& Just 1991).

WM, especially PSTM, has therefore been very clearly found to be central to verbal development for monolingual acquisition, through school and into adulthood, particularly when using language in ways that require explicit and/or metalinguistic verbal knowledge, such as reading, vocabulary and analysing complex structures. However, as mentioned above, the assumption currently is that EWM has little significant part to play in normal development of everyday monolingual grammatical language, especially for pre-school children. There has been little research into young children's language - partly due to inherent problems in constructing reliable tests for pre-literate participants, and partly 
because theoretically, EWM control has not been assumed to relate to normal grammatical language - it would be simply impossible to manage normal fluent speech if all grammatical information had to be encoded consciously.

However, for people learning a second language, many of these assumptions need testing and challenging, and linking to a coherent model of how long and short-term memory are used in SLA (Juffs \& Harrington, 2011). As mentioned above, a common assumption in current cognitive models of language (DeKeyser, 1995, 2003; R. Ellis, 2008; Housen et al., 2012; Ullman, 2004) makes a distinction between implicit or procedural knowledge, and explicit or declarative knowledge. Implicit learning, where conscious awareness is not paid to the input, is associated with child second language acquisition and naturalistic second language learners (especially low literate learners - Craats et al., 2006; Tarone \& Bigelow 2005). It is argued that WM, for these kinds of language learners, as in young monolingual infants, does not play a major role in implicit acquisition of grammar (Juffs \& Rodriguez 2006). This is most famously expressed in Newport's (1990) "less is more" hypothesis, where younger children's lower levels of WM entail greater implicit learning of grammar. By comparison, post-puberty children and adults' greater WM capacities could override implicit mechanisms, in favour of explicit mechanisms such as use of longer memorised chunks and metalinguistic problem solving. Evidence that SLA, in the familiar setting of the instructed foreign language classroom, may often consist of explicit memorisation and problem solving, can therefore lead to the claim that WM is the "key" to L2 development (Miyake \& Friedman 1998).

To date, most WM research has focused on adult SLA (see the very helpful and clear summary in Juffs \& Harrington 2011), but first I turn to WM research on child learners of an L2.

\section{Child SLA and WM}

How early and how widely can we see WM effects in L2 learners? Following the research into PSTM effects in monolingual child development, it was logical for early research in SLA to start by investigating PSTM effects in L2 acquisition. In these early studies, it was found that WM, particularly PSTM, predicted L2 proficiency across a range of measures of vocabulary and grammar (Ando et al., 1992; Cheung, 1996; Service, 1992; Service \& Kohonen, 1995). More recent research (e.g. French, 2006; Masoura and Gathercole, 2005) 
confirmed these strong PSTM effects on L2 proficiency. Similarly to Mitchell et al in this volume, these studies indicated threshold effects of PSTM in less proficient learners compared to more proficient learners - for example in Cheung's (1996) study of primary age Hong Kong children, only participants with a below-mean vocabulary knowledge showed PSTM effects on their L2 vocabulary learning.

Closer inspection of the methodologies used in some of these studies suggests the claim that PSTM supports general L2 development in child SLA may be problematic. The effects of PSTM on vocabulary are indeed robust; however, it seems, especially in Ando et al. (1992) and Service (1992), that the grammar tests used in these studies were more lexical than purely grammatical, and involved explicit metalinguistic knowledge rather than requiring implicit rule-based knowledge. Therefore success on these grammar tests would therefore be likely to be confounded by lexical knowledge rather than be a reflection of PSTM effects.

French and O'Brien (2008) specifically aimed to unpick the potential confound between lexical knowledge and grammatical learning, in their study of L2 French learners of English, aged around 11 years old, over an intensive learning programme of 5 months. Their careful statistically rigorous research was able to identify a clear role for PSTM in learning grammatical knowledge, showing that PSTM scores, measured at the start of the study accounted for nearly $30 \%$ of significant variance on the grammar scores, "after taking into account L2 contact, nonverbal ability, earlier grammar skill, and most importantly, vocabulary knowledge" (2008: 476).

Therefore, it does seem possible to claim that PSTM does indeed predict, or at least, mediate L2 learning in child SLA where the emphasis is on vocabulary or lexically-driven grammar learning. It is less clear that EWM plays a role in complex language processing, as existing research shows contradictory findings, although there are very few studies using EWM specifically rather than PSTM.

One problem in comparing studies of EWM effects in child SLA, especially in processing complex grammar, is that so many aspects of language and cognition are still developing in child learners (Luciana et al, 2005). First language grammatical development of complex structures such as relative clauses and passives are known to be later acquired around the age of seven to nine years; other aspects of sociolinguistic, pragmatic and metalinguistic 
knowledge of language at discourse-level may also not be fully developed until the teenage years (Karmiloff-Smith, 1986). WM capacity, as we saw earlier, is expected to increase up to a steady plateau until aged around 16. Given the additional cognitive load on bilingual use of language (Paradis, 2004), it is therefore not surprising to see varied findings in child SLA research. As learners reach the steady stage of WM capacity around 16 years, it ought to be possible to establish clear robust tasks to measure WM in different L2 populations, and for different language purposes. So I turn now to offer a brief review of the research to date on WM effects on adult learners of a second language.

\section{Adult SLA and WM}

Even in adult SLA, there remain many problems in establishing clear WM effects. These problems are often due to variability in WM methodology (Juffs \& Harringon 2011). This variability then conflicts with the wide variety of stages of development in L2 among adult learners, from those who may have started learning the L2 at primary school to college $a b$ initio learners. In addition there is the question of researching WM effects in adults who are already multilingual, or those who may be bi/multilingual but non-literate (Juffs \& Rodriguez 2006), all of which creates different predictions for WM research, by broadening out the learnability implications beyond the standard instructed foreign language context.

There is also of course the rarely considered question of age-related effects of WM on language learning and development, as WM and cognitive capacity generally, starts to decline beyond the mid-adult plateau. There is widely cited research on the benefits of existing bilingualism to offset aging effects on cognitive performance (e.g. Bialystok et al., 2004). This is usually ascribed to the beneficial effects on executive function arising from the demands of controlling two or more languages. However, it remains to be seen how PSTM or EWM, rather than general measures of executive function, may interact with aging in bilinguals (Luo et al., 2013). Furthermore, there is virtually no research on the impact of PSTM/EWM in older beginner learners of languages, who are often invisible in SLA research (Mackey \& Sachs, 2012).

Despite so much variability in WM and L2 measures, some studies have found clear effects for PSTM and EWM in adult learners, as for younger learners. The two main claims from the monolingual and child L2 research, discussed above, are that PSTM is involved in learning 
novel verbal material, and therefore will aid lexical learning and some aspects of grammatical rule-based learning; second, that WM supports complex management of verbal input and retrieval and will therefore aid sentence processing, reading comprehension, and general language fluency, either in written or spoken form. A third strand of research suggests that EWM will aid executive control, which could be particularly important as learning progresses, in aiding learners to inhibit the L1 (Bialystok, 2002). Mitchell et al. in this volume have claimed, in line with other classic studies of WM effects in SLA, that PSTM aids less proficient earlier-stage learners, and EWM aids more proficient learners. I here highlight some specific studies that have provided evidence to support or contest these claims and so have important implications for future research into WM in SLA.

The first question is whether PSTM/EWM affects all learners, or has greater effects on earlystage learners. The prediction, based on child studies of acquisition such as Service (1992), was that that PSTM/EWM effects would be evident in early learners, especially PSTM, in view of its role in helping build vocabulary. But it remained an open question as to whether there would be a ceiling effect at a certain point of L2 proficiency, or other threshold. Hummel (2009) studied a group of 77 adult upper-intermediate/advanced learners of English to look for PSTM effects on grammatical, lexical and reading scores in a proficiency test. After dividing out the group into a lower and higher proficiency sub-groups, PSTM emerged as a significant predictor of scores on the proficiency measures, especially for vocabulary, but only for the lower proficiency subgroup. She takes this as evidence to support existing assumptions about the learning benefit of PSTM in younger lower-level learners, but suggests this is due to lower levels of lexical knowledge, while the more advanced learners relied on greater lexical knowledge, combined perhaps with more advanced L1 analytic skills, rather than PSTM to help performance on all the L2 proficiency tasks.

Similarly, O'Brien et al. (2006) tested PSTM effects as part of a range of grammatical and lexical measures in college-age participants, in the context of differing types of exposure (stay-at-home vs. study abroad). They found PSTM to be linked to L2 vocabulary use and narrative production only in less proficient learners; interestingly this effect was regardless of the type of exposure.

By comparison, other studies have also found effects only in higher proficiency groups, such as Kormos and Safar's (2008) study of Hungarian adolescents, at beginner and pre- 
intermediate stages, which found PSTM effects only on the higher level pre-intermediate stages.

Turning now to EWM, the key claim here is that EWM will benefit reading comprehension and sentence processing, most evidently in more advanced learners, in line with the monolingual research referred to earlier. Many of these studies have been referenced throughout this volume, but perhaps the seminal study remains Harrington and Sawyer's seminal (1992) study investigating advanced Japanese learners of English. Using a Reading Span test, they found significant effects for EWM between L2 reading ability, and grammatical proficiency, but not when using simple PSTM storage measures of English digits and words. Other studies have also found studies of beneficial effects of EWM on complex language processing (see, particularly, Miyake and Friedman 1998), although as already noted, results can be very varied (Juffs and Harrington 2011), particularly in less advanced stages of learning.

In recent research, Gilabert and Munoz (2010) report on EWM effects on a range of language proficiency measures for a cohort of 59 college-age learners of English, using Reading Span, and split into higher and lower achievers based on a general test of proficiency (the Oxford Placement Test). They found no EWM effects on general proficiency, nor on grammatical accuracy or complexity in a story retelling task in either group; they did find significant, albeit weak, EWM effects on fluency and lexical diversity in the story retelling task, but only in the higher proficiency group.

It therefore seems that proficiency level is not necessarily a key dividing point between the respective roles of PSTM and EWM (such as Reading Span) as claimed by Mitchell et al. in this volume. However, varying uses of proficiency measures need to be taken into account, as a relative split into lower or higher proficiency sub-groups quickly obscures the actual level of proficiency in the group overall. Therefore, among other considerations, the assessment of proficiency level needs careful attention in future research, to avoid these apparent contradictions and ensure reliable comparability.

As well as proficiency differences, there are other potential confounding factors to be resolved to ensure comparability and reliability in WM studies. One such issue relates to how individual differences in WM may differentiate rate of learning, rather than just stage of 
learning. It is possible that there is an internal threshold effect arising from differing WM capacities, rather than a threshold based on L2 proficiency; this threshold is argued to relate to how learners process L2 input. Sunderman and Kroll (2009) used Reading Span to predict EWM effects on changes in lexical knowledge and processing time in learners of Spanish before and after time studying abroad; they only found beneficial effects for those with higher EWM. Sunderman and Kroll conclude that there might be a kind of internal threshold of EWM capacity which facilitates a quicker learning response to L2 input. Therefore those with higher EWM capacity can benefit from a more intense type of input such as study abroad, while those with lower EWM may well feel generally cognitively overloaded during the added exposure of study abroad, so EWM per se does not make a significant difference. To my knowledge, there is no research testing different levels of PSTM in similar learning contexts using to see if this threshold effect extends to PSTM.

A further question is whether PSTM/EWM effects are task-specific, particularly in relation to WM processing and fluency. Logically, we would expect task performance to be affected by different cognitive loads (Robinson, 2001), in terms of differential levels of control; thus WM could be expected to have a role in more complex cognitive tasks, which would require a greater executive capacity. Analysing WM effects on fluency, Fortkamp (1999) found positive significant effects of EWM on oral fluency in sixteen advanced learners of English using a Speaking Span task. In order to test how far EWM may be task specific, Fortkamp also measured Reading Span and a reading aloud task, and found significant correlations between these two scores, but no cross-correlations between EWM and proficiency between the two modes, speaking and reading. Fortkamp concluded from these data that EWM is task specific (Turner \& Engle, 1989). Ahmadian (2012), studying forty advanced learners of English, pursued specific effects of time on task planning in oral production; he found that accuracy in a speaking task was mediated by EWM, measured by Reading Span, but only for the group allowed time for online planning (Yuan \& Ellis 2003).

These findings seem to echo Robinson's (2002) research into incidental vs. intentional learning; he argues that EWM does play a role in L2 but in a task-specific way, so that correlations would be conditioned by task complexity and processing mode. This task effect would not logically be expected to relate to PSTM, but to my knowledge, there is very little research testing this hypothesis. 
In addition, several studies have failed to find any effect for WM, contradicting the general claims about the benefits of WM on SLA (Miyake and Friedman, 1998). Author (2013), in a longitudinal study of thirty-two Chinese learners of English, found that WM was significantly associated with grammatical development in a question production task, but only in the form of a novel story-recall task (specially designed to combine PSTM and Listening Span), but not for other standard EWM tasks, such as Digits Back or Listening Span. Sagarra (2000) found no effect for EWM using Reading Span on L2, looking at grammatical development for intermediate learners of Spanish. Mizera (2006) also found no relation between EWM and L2, using a variety of measures of fluency in early learners of Spanish.

This brief overview of research into SLA over the lifespan has shown that there are reasonably reliable effects for PSTM on learning, in terms of vocabulary and explicit metalinguistic grammatical knowledge. There is less clear reliable evidence for EWM effects on language processing or learning in more general terms. However, it is debatable that PSTM favours earlier learners per se, while EWM favours more advanced language learners, more that different aspects of WM may interact with different types of learning process, and rate of learning.

It has been seen that the evidence of support for effects of WM remain contradictory, largely due to lack of comparability in methodology in research design. The theoretical basis for the predicted effects of PSTM on lexical development, and of WM on processing and fluency, have not seriously been challenged, and it remains a rich potential area to be validated through developing more robust and reliable methodologies, particularly for older learners, and in a wider range of learning settings. However, there are some theoretical and empirical issues which this overview has touched on, and I discuss them now in more detail.

\section{Theoretical and methodological implications for future WM research}

Some of the difficulties and contradictory findings may arise from the current range of tests used and the differences in participants involved. There are major issues remaining about the age effects at both end of the lifespan, and about specific populations beyond the standard school-age foreign language learner, where we have little or no information about how WM may be expected to affect SLA. These would be fruitful and exciting areas to pursue. 
However, I finish with a caveat about expecting too much from WM tests, span tests such as Daneman and Carpenter's Reading Span Test and its variations for listening and speaking. This has been widely adopted as a suitable measure to test the effects of general WM capacity on proficiency, but may be flawed when being used to predict L2 proficiency, given the lack of robust evidence of benefits for aspects of proficiency, as well as potential task-specific effects noted above (e.g. Mizera, 2006; Sagarra, 2000). In addition, in view of the time lag involved during the test procedure between storage and recall, especially on the longer sentence sets, word recall may in fact be using some other kind of short-term memory rather than the phonological loop (Mizera, 2006: 17). The phonological loop is assumed to be around 1-2 seconds which would not be long enough to hold the information required in the longer sets of sentences. It is possible that successful recall could be tapping into some element of long-term working memory (Ericsson \& Kintsch 1994), or it might be evidence of Baddeley's suggested episodic buffer (Baddeley, 2000), although this is purely speculation. Using measures combining PSTM and listening span, such as a story-recall task (Author 2013), could be one way of establishing a less task-dependent effect. Similarly, using a nonverbal task such as a mathematical task (Turner \& Engle, 1986) would avoid any confound with verbal lexical or analytic skills, rather than WM per se.

One alternative is to pursue the claim that WM is domain-specific (Caplan \& Waters 1999, Luo et al., 2013), especially in terms of executive WM rather than PSTM. Caplan and Waters (1999) follow Shah and Miyake (1996) in fractionating out the processing element of WM (the central executive) into verbal and visual components, and then into further subfractioned divisions between different types of verbal processing. Caplan and Waters suggest that the "interpretive processing" system (1999: 78), used for assigning syntactic structure and meaning to a sentence, is different to other verbal WM systems as used for long term storage, planning actions and other "post-interpretive" processing. This approach has been used in a few studies (e.g. for L2, Sunderman and Kroll 2009), but has not been central to much of the research in language processing and WM.

It is easy to fall back on the typical short-hand view of WM, especially PSTM, as a part of the memory system, acting as a bottle-neck to learning by limiting how much we can consciously notice and process at the same time. But we need to move on from this simplistic model. Miyake and Shah (1999: 445) concluded that WM should not be considered as a separate "box" for short term storage that is structurally distinct from other memory systems, 
but more as a set of processes that "control" cognitive actions, and this should now include a discussion of procedural WM (Oberauer, 2010). We also need to articulate better how WM and long-term memory (LTM) intersect in language learning and use, especially in retrieving linguistic knowledge in online performance, perhaps through a better integration of Ericsson and Kintsch's (1995) construct of LT-WM, and Cowan's (2005) view of WM as "temporarily accessible" LTM. As yet, these psychological models, usually based on monolingual children or adults, do not always easily translate into linguistic models of language knowledge, storage, retrieval and processing which are relevant for SLA. Psychologists, linguists, language teachers and language learners all need to keep talking to each other to help tease out where the field will go from here.

\section{Conclusion}

It is clear that we are just at the start of beginning to understand how WM may or may not work in SLA across the lifespan. The priority is for greater clarity and reliability in WM methodology to distinguish between PSTM and EWM, and how the different roles of these constructs for storage, processing and control could be predicted to work in SLA. We need to clarify how best to test WM in different SLA populations at different stages of the lifespan. We must also specify more clearly what aspects of SLA we can logically expect to be supported by WM, given the recurring debates over the nature of SLA in terms of explicit vs. implicit learning (N. Ellis 2005, Han \& Finneran 2013), or knowledge vs. performance (Housen et al., 2012), or as skill-development (DeKeyser, 2003). This need for clarity is an inevitable product of a fertile cross-disciplinary overlap between linguistics, psychology and education, among other fields. But it does require care on the part of SLA researchers in what we can predict and test reliably.

There are still therefore many gaps in the theoretical and empirical literature to help us understand the role of WM in SLA across the life-span. This book is a very welcome addition to the field, and it is hoped that many more studies will be inspired by the research described here to take this field forward. 